Review Article

\title{
Noncoding RNAs in Unexplained Recurrent Spontaneous Abortions and Their Diagnostic Potential
}

\author{
Ningyi Jia $(\mathbb{D})$ and Jian Li $(\mathbb{D}$ \\ Beijing Obstetrics and Gynecology Hospital, Capital Medical University, Beijing, China \\ Correspondence should be addressed to Jian Li; lijian571015@163.com
}

Received 7 August 2019; Revised 19 November 2019; Accepted 25 November 2019; Published 4 December 2019

Academic Editor: Chiara Nicolazzo

Copyright (c) 2019 Ningyi Jia and Jian Li. This is an open access article distributed under the Creative Commons Attribution License, which permits unrestricted use, distribution, and reproduction in any medium, provided the original work is properly cited.

Unexplained recurrent spontaneous abortion (URSA) is defined as the loss of two or more consecutive pregnancies before the 20th week of gestation with normal findings on routine screening tests. Our understanding of the cellular and molecular pathogenesis of URSA is still far from complete. Noncoding RNAs (ncRNAs) play a pivotal role in transcription and expression. The functions of ncRNAs may also improve understanding of URSA pathogenesis. Because of their stability in the circulatory system and at the maternal-fetal interface, it may be possible to use ncRNAs as biomarkers for certain disease states. Here, we provide a narrative review of the current state of knowledge about ncRNAs associated with URSA. The possibility of developing a diagnostic tool using ncRNAs is discussed. The underlying mechanisms of how ncRNAs may lead to the onset of URSA are explored in this review.

\section{Introduction}

Unexplained recurrent spontaneous abortion (URSA) is defined as the loss of two or more consecutive pregnancies before the 20th week of gestation with normal findings on routine screening tests (such as normal parental karyotypes, endocrine, infection, and immune parameters) [1]. URSA accounts for $15 \%$ of recurrent spontaneous abortions [2]. The etiology of URSA remains poorly defined owing to it being identified by a diagnosis of exclusion and its complex molecular and cellular regulation. It is suggested that aberrant gene expression is a major cause of URSA and an important indicator of pregnancy disorders $[3,4]$. ncRNAs are important regulators of transcription and protein expression [5]. The dysregulation of ncRNAs may be involved in the etiology of many pregnancy-related disorders, such as URSA. In biomedical research, ncRNAs are gaining increasingly greater importance as novel biomarkers for diagnosis, prediction, prognosis, and reaction to therapy in diseases. Apparently, ncRNA expression profiling is more effective than mRNA expression profiling for differentiating between normal and pathological tissues [6]. Therefore, the link between URSA and ncRNAs may offer new insights into the pathogenesis and clinical diagnosis of this disease.

Noncoding RNA (ncRNA) can be classified based on its length. One class consists of small transcripts less than 200 nucleotides long. (This class includes microRNAs (miRNAs).) The other class consists of long noncoding RNAs (lncRNAs), which have a length of no less than 200 nucleotides [7]. There are some other kinds of ncRNAs such as circular RNA (circRNA), small nucleolar RNA (snoRNA), piwi-interactive RNA (piRNA), and small nuclear RNA (snRNA) [8]. Considering the wide range of findings published on miRNA and IncRNA, we focus on these two ncRNAs in our review.

Each type of ncRNA has distinct roles in various physiological and pathophysiological processes. With a length of 18-25 nucleotides, miRNA is the dynamic regulator of gene expression [9]. miRNAs perform their functions as part of the RNA-induced silencing complex, which is capable of recruiting target mRNA and initiating translation inhibition or mRNA degradation [10] (Figure 1). CircRNAs can serve as miRNA sponges by binding to proteins to regulate the expression of target genes at the transcriptional level [11]. In recent years, reports have shown that lncRNAs play an 


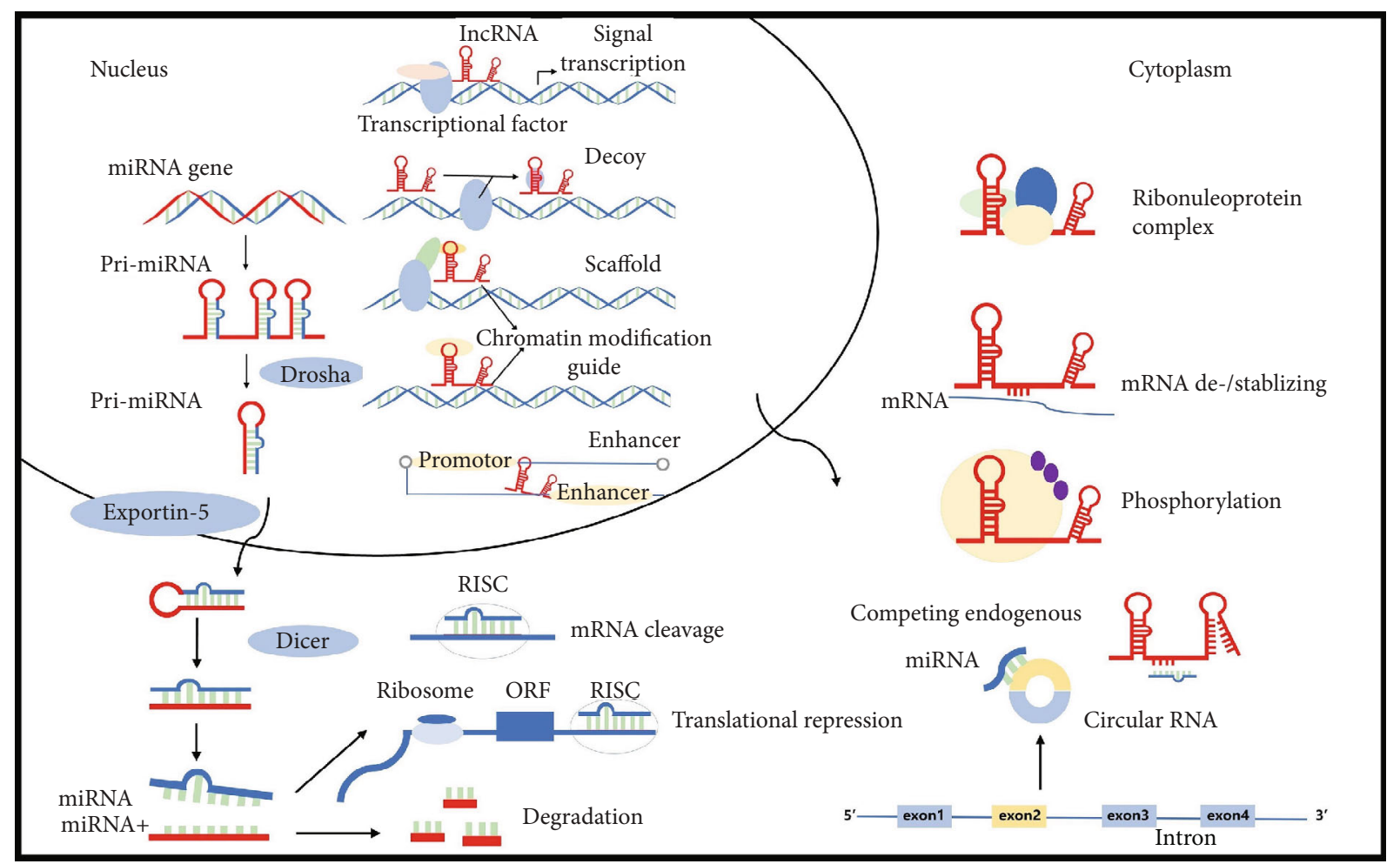

Figure 1: The functions of miRNA and lncRNA and the interaction of ncRNAs. Pre-miRNA is transported into the cytoplasm through exportin-5 and Ran-GTP where it is cleaved by Dicer and finally converted into mature miRNA. This double-stranded small RNA separates into two single strands. One strand from mature miRNA is loaded into the RISC that recruits target mRNA and initiates either translation inhibition or mRNA degradation. lncRNA acts as scaffolds, signals, decoys, guides, miRNA sponges, and enhancer RNAs. They can also contribute to the stability of mRNA. They can serve as downstream regulators of miRNA by acting as competing endogenous RNAs. circRNAs act as miRNA sponges or competing endogenous RNA.

important role in chromatin modification, transcription, translation, gene imprinting, regulation of protein bioactivity, and alternative splicing $[12,13]$. They act as scaffolds, signals, decoys, guides, miRNA sponges, and enhancer RNAs. Proteins and other RNAs can be bound by scaffold lncRNAs to form larger functional complexes. IncRNAs can act as signals to regulate transcription. Decoy lncRNAs are able to block the normal function of target proteins by binding to specific domains. They can also serve as downstream regulators of miRNA by acting as competing endogenous RNAs $[14,15]$. In addition, small regulatory RNAs such as miRNAs can be bound and sequestered by IncRNAs [16], while enhancer RNAs play an important role in the regulation of the expression of neighboring genes. These ncRNAs may have clinical and pathological connections with human diseases.

The genesis and development of the placenta are closely related to the proliferation, differentiation, and functional status of trophoblast cells. It is generally believed that trophoblast cell dysfunction mediates pregnancy disorders [17]. Previous studies have shown that ncRNAs are involved in several steps of the implantation process. Certain ncRNAs have been shown to significantly contribute to the dysfunction of trophoblasts in pregnancy-related disorders [18-21]. Moreover, many publications have reported that the failure of maternal-fetal immunological tolerance and responses can contribute to the pathogenesis of URSA [22-25]. Therefore, identifying certain molecules, as well as their roles in the maternal-fetal interface and peripheral blood, will enable us to have a more comprehensive understanding of the pathogenesis of URSA. The abnormal expression of certain ncRNAs may provide new insights into potential biomarkers for URSA. We focus on ncRNAs in URSA patients in this review.

\section{2. ncRNA Expression Profiling in URSA}

Abnormal expression of ncRNA at the maternal-fetal interface has been observed in URSA (Table 1). To measure differential expression of ncRNA in a specific morbid state, a widely used approach is genome-wide ncRNA profiling by either microarray or RNA-sequencing, followed by reverse transcription quantitative PCR (RT-PCR) [26]. Since ncRNAs are involved in many biological processes, the change in the level of ncRNAs at the maternal-fetal interface may be associated with URSA pathogenesis.

Dong et al. used a miRNA microarray to profile differentially expressed miRNAs in villi and decidual cells isolated from a URSA sample and a control sample. A total of $50 \mathrm{miR}$ NAs were found to be differentially expressed in villi, and seven miRNAs were found upregulated in the decidua. Gene ontology (GO) analysis showed that the most significantly 
TABLE 1: ncRNA expression profiles in the maternal-fetal interface of URSA.

\begin{tabular}{|c|c|c|c|c|c|}
\hline Reference & Microarray filtering criteria & Method & Sample & Upregulated & Downregulated \\
\hline [27] & $q$ value $\leq 0.05 ; \mathrm{FC} \geq 2$ or $\leq 0.5$ & $\begin{array}{l}\text { Microarray } \\
\text { qRT-PCR }\end{array}$ & $\begin{array}{c}\text { Villi } \\
\text { Decidua }\end{array}$ & $\begin{array}{l}4 \text { miRNAs } \\
7 \text { miRNAs }\end{array}$ & $\begin{array}{c}41 \text { miRNAs } \\
\text { Not mentioned }\end{array}$ \\
\hline [28] & $\mathrm{FC} \geq 2.0 ; p$ value $\leq 0.05$ & $\begin{array}{c}\text { Microarray } \\
\text { qRT-PCR }\end{array}$ & Villi & 98 miRNAs & 57 miRNAs \\
\hline [31] & FDR $<0.05 ; p$ value $<0.005$ & Microarray & Decidua & 49 miRNAs & 1 miRNA \\
\hline [32] & $\mathrm{FC}>2.0 ; p$ value $<0.05$ & $\begin{array}{l}\text { RNA-seq } \\
\text { qRT-PCR }\end{array}$ & $\begin{array}{l}\text { Decidua } \\
\text { Villi }\end{array}$ & $\begin{array}{l}23 \text { miRNAs } \\
4 \text { miRNAs }\end{array}$ & $\begin{array}{l}\text { Not mentioned } \\
5 \text { miRNAs }\end{array}$ \\
\hline [33] & $\mathrm{FC}>2.0$ & $\begin{array}{l}\text { Microarray } \\
\text { qRT-PCR }\end{array}$ & Decidua & 859 lncRNAs & $940 \operatorname{lncRNAs}$ \\
\hline$[34]$ & $\mathrm{FC}>2.0$ & $\begin{array}{l}\text { Microarray } \\
\text { qRT-PCR }\end{array}$ & Villi & 467 lncRNAs & $982 \operatorname{lncRNAs}$ \\
\hline [35] & $\mathrm{FC} \geq 2.0 ; p$ value $\leq 0.05$ & $\begin{array}{c}\text { Microarray } \\
\text { qRT-PCR }\end{array}$ & Villi & 335 circRNAs & 259 circRNAs \\
\hline
\end{tabular}

FC: fold change; FDR: false discovery rate.

expressed miRNAs with a fold change of greater than 5 or less than 0.2 were involved in several biological processes, such as regulation of transcription and protein amino acid phosphorylation, whereas a Kyoto Encyclopedia of Genes and Genomes (KEGG) pathway analysis indicated that the predicted target genes were linked to adherence junctions, apoptosis, and the T cell receptor signaling pathway [27]. Specifically, according to the KEGG pathway analysis, the target genes of those miRNAs were deeply involved in the mitogen-activated protein kinase (MAPK) pathway, B cell receptor, and T cell receptor signaling pathways during the development of URSA [28]. The MAPK signaling pathway is of great importance in maintaining normal pregnancy processes, including embryonic development, differentiation of trophoblast cells, and vascular endothelial cell proliferation and differentiation $[29,30]$. Two more studies also illustrated the relationships between target genes and signaling pathways $[31,32]$. Wang et al. reported that predicted target genes participate in programmed cell death, apoptosis, and cell proliferation in the decidua. The major pathways included ErbB signaling, focal adhesion, p53-signaling, and apoptosis. In villi, target genes are widely involved in the regulation of cell proliferation, apoptosis, and angiogenesis. The KEGG pathway analysis revealed that the relevant pathways were apoptosis, p53-signaling, and the cell cycle [32].

A study on genome-wide differential expression of IncRNA showed that, predominantly, infection and inflammation pathways are the ones altered in URSA [33]. Wang et al. imply that the differentially expressed lncRNAs are involved in pathways related to immunity. Furthermore, lncRNA-regulated genes are believed to be important regulators of immune cell activation and differentiation. It is suggested that lncRNAs contribute to the pathogenesis of URSA through immunity pathways, which might represent a new insight to the relationship between immunity and URSA [34].

CircRNAs are a recently discovered group of ncRNA, characterized by a circular covalently closed structure. The functions of circRNAs include acting as miRNA sponges or competing with endogenous RNA, regulating gene transcription, and interacting with RNA-binding proteins (RBP) [11]. The sequestration of miRNA-binding sites by circRNA weakens the inhibitory effects of miRNAs on gene expression. A circRNA microarray analysis identified aberrantly expressed circRNAs in the villi of patients with URSA. This may shed light on the role of circRNAs in the onset of URSA [35]. The circRNA microarray revealed miRNA-binding sites on many circRNAs. Downregulation of the miRNAs miR520f [27] and miR-181d [28], which have relevant binding sites on upregulated circRNAs, in URSA dNK cells may contribute to the inhibition of adhesion and cell proliferation [35]. However, the roles of circular RNA and competing endogenous RNA networks remain to be investigated.

The above-mentioned studies strongly suggest that ncRNA dysregulation is closely linked to URSA, in which differentially expressed ncRNAs might play a role in pathogenesis.

\section{Structural Changes in ncRNAs in URSA}

The change of ncRNA structure may also contribute to the pathogenesis of URSA. It is believed that single nucleotide polymorphisms (SNPs) in miRNAs could influence biological processes by changing miRNA target selection and contribute to the occurrence of diseases [36]. It has been reported that the miRNA miR-423 may be implicated in the genetic predisposition to URSA because it disrupts the production of mature miR-423 and its target PA2G4 [37]. Furthermore, the miRNA miR-10a impairs the expression of Bim, which affects URSA progression [38]. Also, in the coding region of miR-323b, the SNP rs56103835 T>C was associated with URSA. The expression of miR-323b was elevated in cell lines transfected with the T-C haplotype. The T-C haplotype can inhibit proliferation and migration and promote apoptosis in HTR-8/SVneo cells [39]. One nucleotide mutation in the coding region of pri-miR-125a was associated with the onset of URSA, which was due to two polymorphisms found in patients. The $A>G$ mutation 
reduced miR-125a expression. Mutant pri-miR-125a can enhance endometrial stromal cell (ESC) invasiveness and disturb the expression of the miR-125a targetome, which influences cell proliferation, migration, invasion, and embryonic development [40, 41]. It was suggested that miR196a2CC, miR-499AG+GG, and the miR-196a2CC/miR499AG+GG combination are associated with URSA [42]. The variants of miR-27a also play a pivotal role in predicting folate levels in URSA patients [43].

\section{4. ncRNA May Contribute to the Pathogenesis of URSA}

Studies suggested that abnormal expression of ncRNAs can result in disorders of pregnancy. Wang et al. [44] provide evidence that miR-133a was strongly overexpressed in URSA villi. The following findings confirm that miR-133a decreases expression of histocompatibility antigen, class I, G (HLA-G), which can lead to impairment of decidual NK cell invasion and angiogenesis [45]. The change of miR-27a-3p, miR-29a-3p, miR-100-5p, miR-127-3p, and miR-486-5p levels in villi can lead to the abnormity of invasiveness in URSA [46]. In another study, it was found that overexpressed miR-520 in the villi of URSA patients was correlated with trophoblast cell apoptosis via suppression of PARP1 expression [47]. Zhu et al. found that miR16 expression was significantly upregulated in the decidua and villi of URSA patients. It was suggested that miR-16 regulates placental angiogenesis by influencing VEGF expression, which may contribute to the pathogenesis of URSA [48]. It has been verified that compared to normal mice, miR-3074-5p expression was significantly upregulated and miR-486-3p expression was significantly downregulated in mice with URSA [49]. The later research provided evidence that miR-3074-5p promotes the apoptosis but inhibits the invasiveness of HTR8/SVneo cells in vitro [50].

Studies show that the IncRNA HOTAIR can be inhibited in URSA, which can influence trophoblast invasion and the expression of Matrix Metallopeptidase 9 (MMP9) [51, 52]. Moreover, Lnc-SLC4A1-1 can recruit nuclear factor kappalight-chain-enhancer of activated B cells (NF- $\kappa$ B) and ultimately upregulate the expression of interleukin 8 (CXCL8). This in turn elevates the levels of tumor necrosis factor alpha (TNF- $\alpha$ ) and interleukin 1 beta (IL-1 $\beta$ ) and affects trophoblast function. Dysfunction of Lnc-SLC4A1-1 may affect immune response and lead to URSA [53].

Researchers have found that miRNAs can influence the balances of TH1/TH2 and TH17/Treg cells in many pregnancy complications $[54,55]$. Recent studies reveal that miRNAs participate in the establishment of immune tolerance at conception and may contribute to the regulation of dendritic cells and $\mathrm{T}$ regulatory cells generated by seminal fluid [56]. The miR-106b-25 93 cluster, recognized as Treg-associated miRNAs, showed a higher expression in URSA patients. An imbalanced immune response and dysregulated $\mathrm{T}$ cell function may lead to reproductive failure. Immune cell-related miRNA profiles may serve as biomarkers and be used for making prognoses during treatment of URSA patients [55]. As is mentioned above, miR133a in the villi can lead to downregulation of HLA-G expression and impairment of angiogenesis and invasion [45]. These immune abnormalities generate an inflammatory response, thus affecting function at the maternal-fetal interface (Figure 2).

\section{5. ncRNA as a Potential Diagnostic Tool for URSA}

Although it has been verified that ncRNA expression profiles are different in different disease states, obtaining biopsy samples from organs for pathological profiling is unrealistic. Samples obtained from the circulation offer an ideal alternative. The majority of circulating miRNAs are associated with protein complexes or high-density lipoprotein [57, 58]. Meanwhile, miRNAs may also be found encapsulated within cellular vesicles $[59,60]$. The "packaging" of miRNAs contributes to their stability in plasma. Owing to their stability in blood, ncRNAs have been explored as biomarkers for many disease states. The differential expression of ncRNA has been reported in cancers [61], cardiac disease [6], and some pregnancy disorders such as preeclampsia [62-64]. The predictive potential of these ncRNAs as early indicators of disease is highlighted in this review.

Published studies have reported abnormal expression of some miRNAs in maternal circulation. Although these studies put forth the idea that these circulating miRNAs might be biomarkers of URSA, in some of them, a receiver operating characteristic (ROC) curve has not been performed and the results need to be validated in an independent cohort. Yang et al. suggest that levels of miR-127a-3p and miR-486-5p in plasma could serve as predictive factors for URSA [46]. Qin et al. found four circulating miRNAs that were upregulated and one circulating miRNA that was downregulated that demonstrated potential as biomarkers for URSA [65]. miRNAs play a pivotal role in peripheral blood monocular cells (PBMCs), in regulation and differentiation of T cells. In PBMCs, miR-25, miR-93, and miR-106b were found to be elevated while miR-146-a and miR-155 were downregulated [55]. Several studies have found that in maternal circulation, mutated miRNAs may be associated with URSA. However, the use of ncRNAs as noninvasive diagnostic biomarkers has not yet been established (Figure 2). Thus, studies on larger populations are needed in order to validate the potential of ncRNAs.

ncRNAs could potentially be used for the treatment of pregnancy complications in the future. A single ncRNA may affect multiple cellular pathways. ncRNAs are shed from primary cells into the circulation and are involved in cell-tocell communication. The targeting of a single ncRNA may affect multiple downstream pathways. Recently, many different methods of silencing lncRNAs in cancer have been reported. However, targeting pathways may result in some side effects. From what has been discussed above, the functions of ncRNAs should be better understood before they are used as therapeutic targets.

There are no commonly accepted methods for diagnosis of URSA nor any efficient treatments, due to the lack of studies with abundant sample size, independent validation cohorts, or analysis of correlation with disease characteristics. 


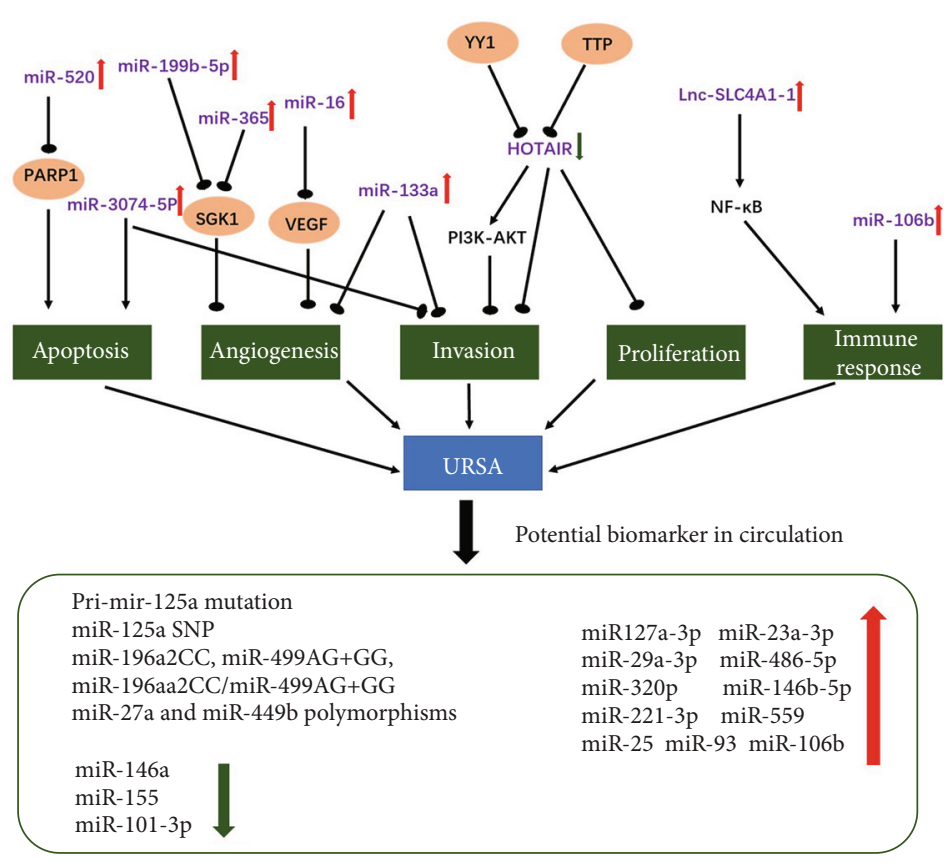

FIGURE 2: The dysregulation of ncRNAs and their function in the pathogenesis of URSA. The potential diagnostic ncRNAs in circulation in URSA patients.

Major gaps in knowledge need to be filled before findings can be translated into clinical applications.

\section{Conclusion}

URSA is not a rare disease, but its molecular and cellular pathogenesis remains poorly understood. Emerging evidence shows that the expression profiles of ncRNAs at the maternal-fetal interface and in the circulation of URSA patients have a biological relevance, allowing for a better understanding of the disease. Through microarray and RTPCR assays, some dysregulated ncRNAs in both URSA cells and tissues have been identified. A growing number of studies have suggested functional roles for ncRNAs in URSA and provided new clues for their clinical application as potential biomarkers. However, functional studies of these ncRNAs are still limited. Existing research can provide us some insight into the pathogenesis of URSA.

Although many studies have demonstrated that ncRNAs are critical in placental development, differences exist between animals and humans in placental development and placental ncRNA expression profiles. Many studies of ncRNAs in placenta were performed using cell lines, which involve different conditions compared to experiments performed in vivo. The roles of ncRNAs in the pathogenesis of URSA are still far from being understood, especially when compared with the progress made in other disease fields. Further investigation of the contributions of ncRNAs for modulating cellular activities in URSA is required.

\section{Conflicts of Interest}

The authors have no conflict of interest relevant to this study.

\section{Authors' Contributions}

Ningyi Jia prepared the manuscript. Jian Li provided the outlines. All authors read and approved the final manuscript.

\section{References}

[1] T. C. Li, M. Makris, M. Tomsu, E. Tuckerman, and S. Laird, "Recurrent miscarriage: aetiology, management and prognosis," Human Reproduction Update, vol. 8, no. 5, pp. 463-481, 2002.

[2] X. Santamaria and H. Taylor, "MicroRNA and gynecological reproductive diseases," Fertility and Sterility, vol. 101, no. 6, pp. 1545-1551, 2014.

[3] O. Brew, M. H. Sullivan, and A. Woodman, "Comparison of normal and pre-eclamptic placental gene expression: a systematic review with meta-analysis," PLoS One, vol. 11, no. 8, article e0161504, 2016.

[4] A. Houshmand-Oeregaard, L. Hjort, L. Kelstrup et al., "DNA methylation and gene expression of TXNIP in adult offspring of women with diabetes in pregnancy," PLoS One, vol. 12, no. 10, article e0187038, 2017.

[5] R. P. Alexander, G. Fang, J. Rozowsky, M. Snyder, and M. B. Gerstein, "Annotating non-coding regions of the genome," Nature Reviews. Genetics, vol. 11, no. 8, pp. 559-571, 2010.

[6] J. Viereck and T. Thum, "Circulating noncoding RNAs as biomarkers of cardiovascular disease and injury," Circulation Research, vol. 120, no. 2, pp. 381-399, 2017.

[7] P. Kapranov, J. Cheng, S. Dike et al., "RNA maps reveal new RNA classes and a possible function for pervasive transcription," Science, vol. 316, no. 5830, pp. 1484-1488, 2007.

[8] The ENCODE Project Consortium, "An integrated encyclopedia of DNA elements in the human genome," Nature, vol. 489, no. 7414, pp. 57-74, 2012. 
[9] D. P. Bartel, "MicroRNAs: target recognition and regulatory functions," Cell, vol. 136, no. 2, pp. 215-233, 2009.

[10] E. E. Marsh, Z. Lin, P. Yin, M. Milad, D. Chakravarti, and S. E. Bulun, "Differential expression of microRNA species in human uterine leiomyoma versus normal myometrium," Fertility and Sterility, vol. 89, no. 6, pp. 1771-1776, 2008.

[11] T. B. Hansen, T. I. Jensen, B. H. Clausen et al., "Natural RNA circles function as efficient microRNA sponges," Nature, vol. 495, no. 7441, pp. 384-388, 2013.

[12] J. J. Quinn and H. Y. Chang, "Unique features of long noncoding RNA biogenesis and function," Nature Reviews. Genetics, vol. 17, no. 1, pp. 47-62, 2016.

[13] Y. Wang, Y. Mo, Z. Gong et al., "Circular RNAs in human cancer," Molecular Cancer, vol. 16, no. 1, p. 25, 2017.

[14] T. Hung, Y. Wang, M. F. Lin et al., "Extensive and coordinated transcription of noncoding RNAs within cell-cycle promoters," Nature Genetics, vol. 43, no. 7, pp. 621-629, 2011.

[15] I. Martianov, A. Ramadass, A. Serra Barros, N. Chow, and A. Akoulitchev, "Repression of the human dihydrofolate reductase gene by a non-coding interfering transcript," Nature, vol. 445, no. 7128, pp. 666-670, 2007.

[16] J. M. Franco-Zorrilla, A. Valli, M. Todesco et al., “Target mimicry provides a new mechanism for regulation of microRNA activity," Nature Genetics, vol. 39, no. 8, pp. 1033-1037, 2007.

[17] G. C. S. Smith, "First-trimester determination of complications of late pregnancy," JAMA, vol. 303, no. 6, pp. 561-562, 2010.

[18] X. He, Y. He, B. Xi et al., "LncRNAs expression in preeclampsia placenta reveals the potential role of LncRNAs contributing to preeclampsia pathogenesis," PLoS One, vol. 8, no. 11, article e81437, 2013.

[19] H. Chen, T. Meng, X. Liu et al., "Long non-coding RNA MALAT-1 is downregulated in preeclampsia and regulates proliferation, apoptosis, migration and invasion of JEG-3 trophoblast cells," International Journal of Clinical and Experimental Pathology, vol. 8, no. 10, pp. 12718-12727, 2015.

[20] C. Estella, I. Herrer, J. M. Moreno-Moya et al., "miRNA signature and Dicer requirement during human endometrial stromal decidualization in vitro," PLoS One, vol. 7, no. 7, article e41080, 2012.

[21] K. Qian, L. Hu, H. Chen et al., "Hsa-miR-222 is involved in differentiation of endometrial stromal cells in vitro," Endocrinology, vol. 150, no. 10, pp. 4734-4743, 2009.

[22] L. Wu, L. H. Luo, Y. X. Zhang et al., "Alteration of Th17 and Treg cells in patients with unexplained recurrent spontaneous abortion before and after lymphocyte immunization therapy," Reproductive Biology and Endocrinology, vol. 12, p. 74, 2014.

[23] J. Yuan, J. Li, S. Y. Huang, and X. Sun, "Characterization of the subsets of human NKT-like cells and the expression of Th1/Th2 cytokines in patients with unexplained recurrent spontaneous abortion," Journal of Reproductive Immunology, vol. 110, pp. 81-88, 2015.

[24] P. Dong, X. Wen, J. Liu et al., "Simultaneous detection of decidual Th1/Th2 and NK1/NK2 immunophenotyping in unknown recurrent miscarriage using 8-color flow cytometry with FSC/Vt extended strategy," Bioscience Reports, vol. 37, no. 3, 2017.

[25] O. B. Christiansen, A. M. Nybo Andersen, E. Bosch et al., "Evidence-based investigations and treatments of recurrent pregnancy loss," Fertility and Sterility, vol. 83, no. 4, pp. 821839, 2005.
[26] Z. Li, X. Li, C. Chen et al., "Long non-coding RNAs in nucleus pulposus cell function and intervertebral disc degeneration," Cell Proliferation, vol. 51, no. 5, article e12483, 2018.

[27] F. Dong, Y. Zhang, F. Xia et al., "Genome-wide miRNA profiling of villus and decidua of recurrent spontaneous abortion patients," Reproduction, vol. 148, no. 1, pp. 33-41, 2014.

[28] L. Tang, C. Gao, L. Gao, Y. Cui, and J. Liu, "Expression profile of micro-RNAs and functional annotation analysis of their targets in human chorionic villi from early recurrent miscarriage," Gene, vol. 576, no. 1, Part 2, pp. 366-371, 2016.

[29] M. De Falco, L. Cobellis, D. Giraldi et al., "Expression and distribution of notch protein members in human placenta throughout pregnancy," Placenta, vol. 28, no. 2-3, pp. 118 126, 2007.

[30] V. Fox, P. J. Gokhale, J. R. Walsh, M. Matin, M. Jones, and P. W. Andrews, "Cell-cell signaling through NOTCH regulates human embryonic stem cell proliferation," Stem Cells, vol. 26, no. 3, pp. 715-723, 2008.

[31] D. Li, J. Li, B. Jia, Y. Wang, J. Zhang, and G. Liu, "Genomewide identification of microRNAs in decidual natural killer cells from patients with unexplained recurrent spontaneous abortion," American Journal of Reproductive Immunology, vol. 80, no. 5, article e13052, 2018.

[32] J. M. Wang, Y. Gu, Y. Zhang et al., "Deep-sequencing identification of differentially expressed miRNAs in decidua and villus of recurrent miscarriage patients," Archives of Gynecology and Obstetrics, vol. 293, no. 5, pp. 1125-1135, 2016.

[33] H. Wang, Q. Cao, J. Ge et al., "LncRNA-regulated infection and inflammation pathways associated with pregnancy loss: genome wide differential expression of IncRNAs in early spontaneous abortion," American Journal of Reproductive Immunology, vol. 72, no. 4, pp. 359-375, 2014.

[34] L. Wang, H. Tang, Y. Xiong, and L. Tang, "Differential expression profile of long noncoding RNAs in human chorionic villi of early recurrent miscarriage," Clinica Chimica Acta, vol. 464, pp. 17-23, 2017.

[35] Y. T. Qian, X. Wang, H. J. Ruan et al., "Circular RNAs expressed in chorionic villi are probably involved in the occurrence of recurrent spontaneous abortion," Biomedicine \& Pharmacotherapy, vol. 88, pp. 1154-1162, 2017.

[36] M. A. Saunders, H. Liang, and W. H. Li, "Human polymorphism at microRNAs and microRNA target sites," Proceedings of the National Academy of Sciences of the United States of America, vol. 104, no. 9, pp. 3300-3305, 2007.

[37] X. Su, Y. Hu, Y. Li et al., "The polymorphism of rs6505162 in the miR423 coding region and recurrent pregnancy loss," Reproduction, vol. 150, no. 1, pp. 65-76, 2015.

[38] Y. Li, X. Q. Wang, L. Zhang et al., "A SNP in pri-miR-10a is associated with recurrent spontaneous abortion in a HanChinese population," Oncotarget, vol. 7, no. 7, pp. 82088222, 2016.

[39] X. Q. Wang, Y. Li, X. Su et al., "Haplotype-based association of two SNPs in miR-323b with unexplained recurrent spontaneous abortion in a Chinese Han population," Journal of Cellular Physiology, vol. 233, no. 8, pp. 6001-6017, 2018.

[40] Y. Hu, Z. H. Huo, C. M. Liu et al., "Functional study of one nucleotide mutation in pri-miR-125a coding region which related to recurrent pregnancy loss," PLoS One, vol. 9, no. 12, article e114781, 2014.

[41] Y. Hu, C. M. Liu, L. Qi et al., "Two common SNPs in pri-miR$125 \mathrm{a}$ alter the mature miRNA expression and associate with 
recurrent pregnancy loss in a Han-Chinese population," $R N A$ Biology, vol. 8, no. 5, pp. 861-872, 2011.

[42] Y. J. Jeon, Y. S. Choi, H. Rah et al., "Association study of microRNA polymorphisms with risk of idiopathic recurrent spontaneous abortion in Korean women," Gene, vol. 494, no. 2, pp. 168-173, 2012.

[43] H. Rah, K. W. Chung, K. H. Ko et al., "miR-27a and miR-449b polymorphisms associated with a risk of idiopathic recurrent pregnancy loss," PLoS One, vol. 12, no. 5, article e0177160, 2017.

[44] X. Wang, B. Li, J. Wang et al., "Evidence that miR-133a causes recurrent spontaneous abortion by reducing HLA-G expression," Reproductive Biomedicine Online, vol. 25, no. 4, pp. 415-424, 2012.

[45] W. Guo, L. Fang, B. Li et al., "Decreased human leukocyte antigen-G expression by miR-133a contributes to impairment of proinvasion and proangiogenesis functions of decidual NK cells," Frontiers in Immunology, vol. 8, p. 741, 2017.

[46] Q. Yang, W. W. Gu, Y. Gu et al., "Association of the peripheral blood levels of circulating microRNAs with both recurrent miscarriage and the outcomes of embryo transfer in an in vitro fertilization process," Journal of Translational Medicine, vol. 16, no. 1, p. 186, 2018.

[47] X. Dong, L. Yang, and H. Wang, "miR-520 promotes DNAdamage-induced trophoblast cell apoptosis by targeting PARP1 in recurrent spontaneous abortion (RSA)," Gynecological Endocrinology, vol. 33, no. 4, pp. 274-278, 2017.

[48] Y. Zhu, H. Lu, Z. Huo et al., "MicroRNA-16 inhibits fetomaternal angiogenesis and causes recurrent spontaneous abortion by targeting vascular endothelial growth factor," Scientific Reports, vol. 6, p. 35536, 2016.

[49] Y. Gu, X. Zhang, Q. Yang et al., “Aberrant placental villus expression of miR-486-3p and miR-3074-5p in recurrent miscarriage patients and uterine expression of these microRNAs during early pregnancy in mice," Gynecologic and Obstetric Investigation, vol. 81, no. 2, pp. 112-117, 2016.

[50] Y. Gu, Y. Shi, Q. Yang et al., "miR-3074-5p promotes the apoptosis but inhibits the invasiveness of human extravillous trophoblast-derived HTR8/SVneo cells in vitro," Reproductive Sciences, vol. 25, no. 5, pp. 690-699, 2018.

[51] F. J. Tian, X. Y. He, J. Wang et al., "Elevated tristetraprolin impairs trophoblast invasion in women with recurrent miscarriage by destabilization of HOTAIR," Molecular Therapy Nucleic Acids, vol. 12, pp. 600-609, 2018.

[52] Y. Zhang, F. Jin, X. C. Li et al., "The YY1-HOTAIR-MMP2 signaling axis controls trophoblast invasion at the maternalfetal interface," Molecular Therapy, vol. 25, no. 10, pp. 23942403, 2017.

[53] Z. Huang, G. Du, X. Huang et al., "The enhancer RNA lncSLC4A1-1 epigenetically regulates unexplained recurrent pregnancy loss (URPL) by activating CXCL8 and NF-kB pathway," eBioMedicine, vol. 38, pp. 162-170, 2018.

[54] E. E. Winger, J. L. Reed, and X. Ji, "First-trimester maternal cell microRNA is a superior pregnancy marker to immunological testing for predicting adverse pregnancy outcome," Journal of Reproductive Immunology, vol. 110, pp. 22-35, 2015.

[55] S. Abdolmohammadi Vahid, M. Ghaebi, M. Ahmadi et al., "Altered T-cell subpopulations in recurrent pregnancy loss patients with cellular immune abnormalities," Journal of Cellular Physiology, vol. 234, no. 4, pp. 4924-4933, 2019.
[56] J. E. Schjenken, B. Zhang, H. Y. Chan, D. J. Sharkey, T. Fullston, and S. A. Robertson, "miRNA regulation of immune tolerance in early pregnancy," American Journal of Reproductive Immunology, vol. 75, no. 3, pp. 272-280, 2016.

[57] K. C. Vickers, B. T. Palmisano, B. M. Shoucri, R. D. Shamburek, and A. T. Remaley, "MicroRNAs are transported in plasma and delivered to recipient cells by highdensity lipoproteins," Nature Cell Biology, vol. 13, no. 4, pp. 423-433, 2011.

[58] J. D. Arroyo, J. R. Chevillet, E. M. Kroh et al., “Argonaute 2 complexes carry a population of circulating microRNAs independent of vesicles in human plasma," Proceedings of the National Academy of Sciences of the United States of America, vol. 108, no. 12, pp. 5003-5008, 2011.

[59] H. Valadi, K. Ekstrom, A. Bossios, M. Sjostrand, J. J. Lee, and J. O. Lotvall, "Exosome-mediated transfer of mRNAs and microRNAs is a novel mechanism of genetic exchange between cells," Nature Cell Biology, vol. 9, no. 6, pp. 654-659, 2007.

[60] A. Gallo, M. Tandon, I. Alevizos, and G. G. Illei, “The majority of microRNAs detectable in serum and saliva is concentrated in exosomes," PLoS One, vol. 7, no. 3, article e30679, 2012.

[61] M. G. Schrauder, R. Strick, R. Schulz-Wendtland et al., "Circulating micro-RNAs as potential blood-based markers for early stage breast cancer detection," PLoS One, vol. 7, no. 1, article e29770, 2012.

[62] M. S. Murphy, C. Tayade, and G. N. Smith, "Maternal circulating microRNAs and pre-eclampsia: challenges for diagnostic potential," Molecular Diagnosis \& Therapy, vol. 21, no. 1, pp. 23-30, 2017.

[63] D. Cretoiu, J. Xu, J. Xiao, N. Suciu, and S. M. Cretoiu, "Circulating microRNAs as potential molecular biomarkers in pathophysiological evolution of pregnancy," Disease Markers, vol. 2016, Article ID 3851054, 7 pages, 2016.

[64] M. Jiang, G. E. Lash, X. Zhao, Y. Long, C. Guo, and H. Yang, "CircRNA-0004904, CircRNA-0001855, and PAPP-A: potential novel biomarkers for the prediction of preeclampsia," Cellular Physiology and Biochemistry, vol. 46, no. 6, pp. 2576-2586, 2018.

[65] W. Qin, Y. Tang, N. Yang, X. Wei, and J. Wu, "Potential role of circulating microRNAs as a biomarker for unexplained recurrent spontaneous abortion," Fertility and Sterility, vol. 105, no. 5, pp. 1247-1254.e3, 2016. 


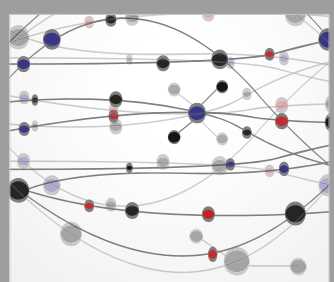

The Scientific World Journal
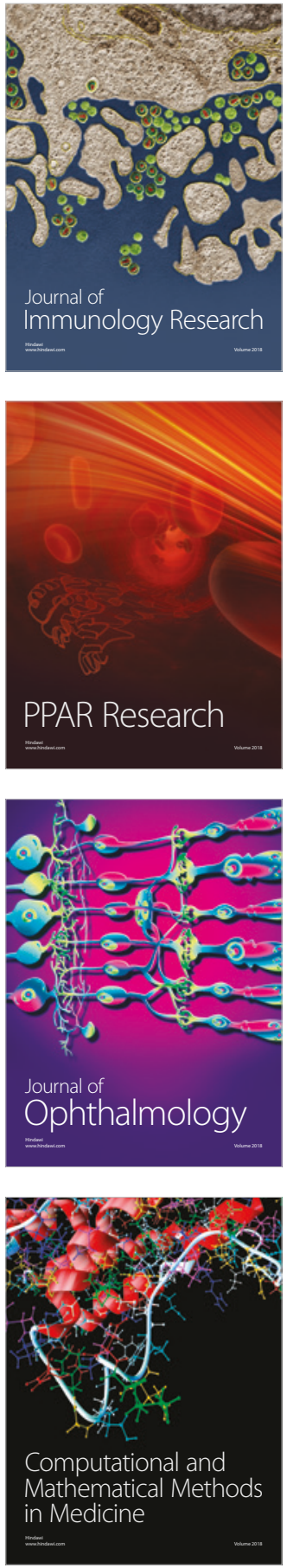

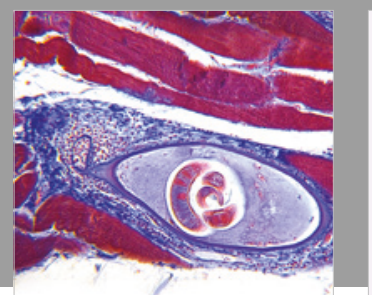

Gastroenterology Research and Practice

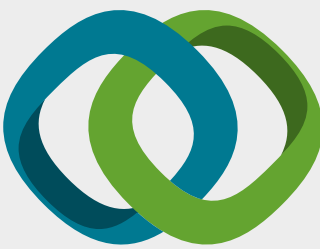

\section{Hindawi}

Submit your manuscripts at

www.hindawi.com
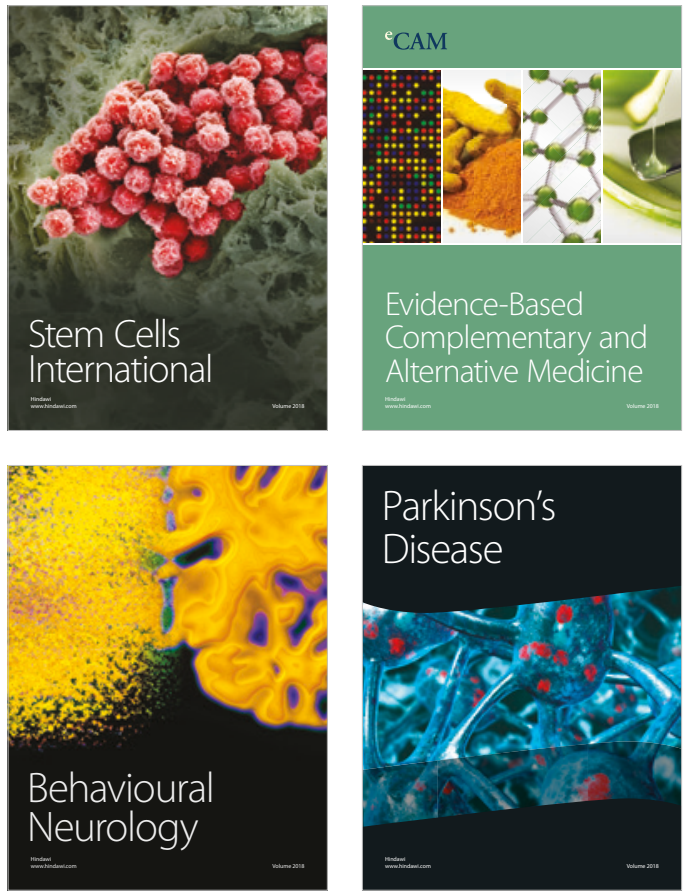

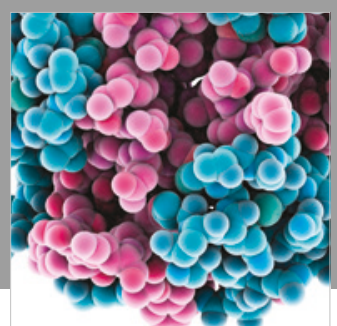

ournal of

Diabetes Research

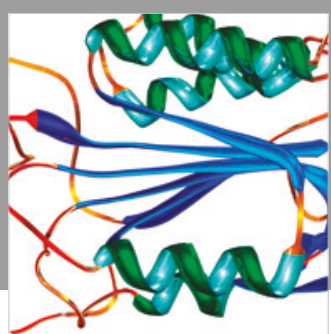

Disease Markers
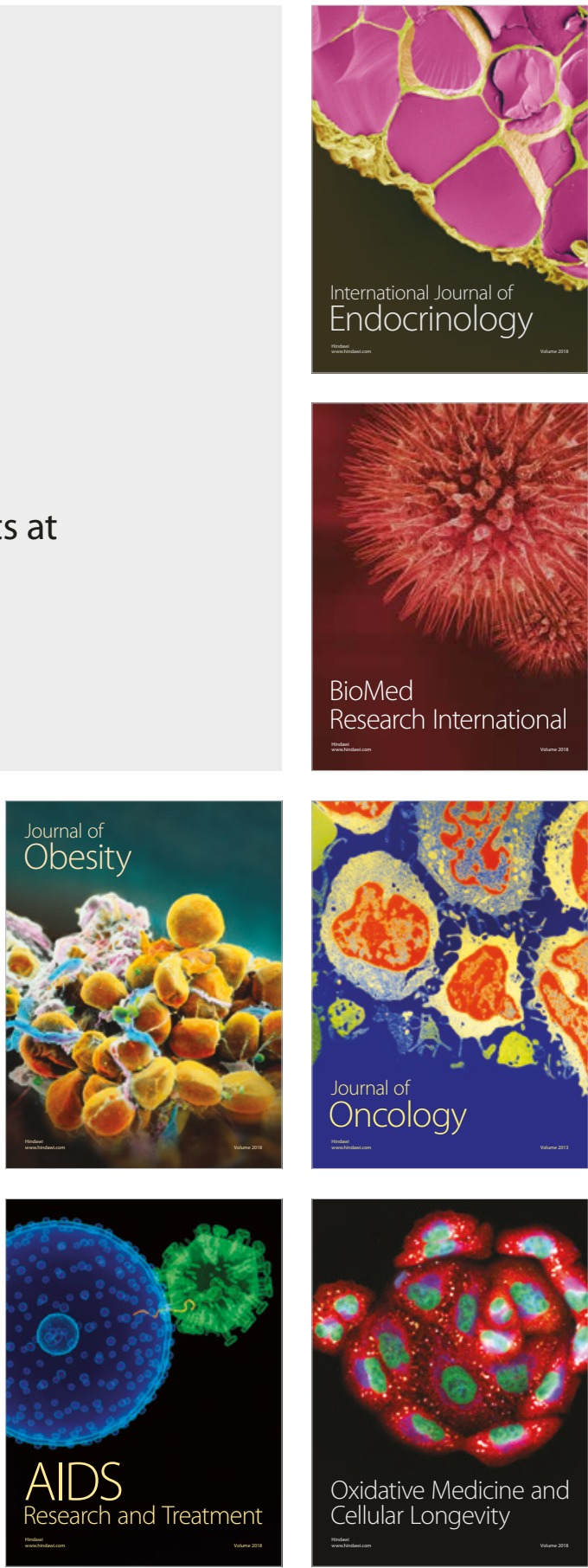